\title{
Influence of Eta-Phase on Wear Behavior of WC-Co Carbides
}

\author{
A. Formisano, F. Capece Minutolo, A. Caraviello, L. Carrino, M. Durante, and A. Langella
}

Department of Chemical, Materials and Production Engineering, University of Naples Federico II, Piazzale Tecchio 80, 80125 Naples, Italy

Correspondence should be addressed to A. Formisano; aformisa@unina.it

Received 30 October 2015; Revised 11 December 2015; Accepted 21 December 2015

Academic Editor: Meng Hua

Copyright (C) 2016 A. Formisano et al. This is an open access article distributed under the Creative Commons Attribution License, which permits unrestricted use, distribution, and reproduction in any medium, provided the original work is properly cited.

\begin{abstract}
Cemented carbides, also known as Widia, are hard metals produced by sintering process and widely used in mechanical machining. They show high cutting capacity and good wear resistance; consequently, they result to be excellent materials for manufacturing cutting tools and sandblast nozzles. In this work, the wear resistance of WC-Co carbides containing Eta-phase, a secondary phase present in the hard metals when a carbon content deficiency occurs, is analyzed. Different mixtures of carbide are prepared and sintered, with different weight percentages of carbon, in order to form Eta-phase and then analyze how the carbon content influences the wear resistance of the material. This characterization is carried out by abrasive wear tests. The test parameters are chosen considering the working conditions of sandblast nozzles. Additional information is gathered through microscopic observations and the evaluation of hardness and microhardness of the different mixtures. The analyses highlight that there is a limit of carbon content below which bad sintering occurs. Considering the mixtures without these sintering problems, they show a wear resistance depending on the size and distribution of the Eta-phase; moreover, the one with high carbon content deficiency shows the best performance.
\end{abstract}

\section{Introduction}

The production of cemented carbides is a fast growing segment in industry and they are used by different marked areas [1]. Tungsten carbide cobalt (WC-Co) based hard metals have both mechanical $[2,3]$ and wear resistance properties [47] better than traditional metals; these characteristics ensure their use for cutting and wear resistant tools [8].

Several studies analyze how the addition of additives to WC-Co, such as $\mathrm{Cr}_{3} \mathrm{C}_{2}$ and VC, influences the wear behavior and the mechanical characteristics of the new sintered materials. In detail, both vanadium and chromium carbide are inhibitors against the grain growth during sintering and increase hardness and toughness; moreover, they influence the friction, improving the wear behavior [9-12].

Conversely, few research studies focus on the wear behavior of WC-Co compounds in the presence of Eta-phase. This secondary phase, a hard and brittle $\mathrm{Co}_{x} \mathrm{~W}_{y} \mathrm{C}$ ternary compound, where $x+y=6\left(\mathrm{M}_{6} \mathrm{C}\right)$ or $12\left(\mathrm{M}_{12} \mathrm{C}\right)$ and $x$ and $y$ can assume integer values in the range of 2 to 6 , is present in cobalt-bonded carbides as a result of decarburizing reactions [13] during sintering or high-temperature chemical vapor deposition (CVD) coating of refractory films on carbide substrates [14]; it can show different microstructure and distribution $[15,16]$ depending on the carbon content and other factors, such as the compacting pressure and the sintering temperature $[17,18]$.

Generally, the formation of Eta-phase is undesirable [19] since its presence causes a detriment of the mechanical properties [20]. Despite this, the literature contains contradictory results relative to the hardness; in fact, this characteristic and then the wear behavior result to be dependent on both grains orientation and applied loads. In this regard, Bonache et al. [21, 22] have highlighted that an accurate observation and characterization of the single phases are of paramount importance in understanding the hard metals behavior.

Consequently, the aim of this paper is to analyze how the presence of Eta-phase influences the properties of wear resistance of WC-Co compounds; the necessity of this analysis resides in the need to study the characteristics of a material usable for the construction of sandblast nozzles. These mechanical components are crossed by a flow of abrasive fluid; then, they are not subject to high loads but must be capable of guaranteeing high resistance to wear. 
TABLE 1: Amount of C on WC and density of the samples.

\begin{tabular}{lcc}
\hline Sample & Amount of C on WC $(\%)$ & Density $\left(\mathrm{g} / \mathrm{cm}^{3}\right)$ \\
\hline A & 6.16 & 15.09 \\
B & 6.05 & 15.16 \\
C & 6.00 & 15.20 \\
D & 5.90 & 15.26 \\
E & 5.70 & 15.05 \\
F & 5.50 & 13.73 \\
\hline
\end{tabular}

For this reason, abrasion tests $[23,24]$ are carried out on WC-Co samples with different carbon deficiency levels, in order to form Eta-phase and to analyze the abrasive wear. Moreover, microscopic observations and hardness and microhardness evaluations are carried out for a macro- and microinterpretation of the abrasion tests data.

The tests furnish information on a technological limit related to this investigation and highlight the differences, in terms of wear properties, associated with the shape and the distribution of Eta-phase.

\section{Materials and Methods}

Specimens from six different samples are prepared at Nashira Hardmetals Company, starting from a powder constituted by 95.5\% of WC and $4.5 \%$ of Co and with an average diameter of $1.5 \mu \mathrm{m}$. The reference sample has the stoichiometric amount of $\mathrm{C}$ in the WC that is $6.16 \%$. The other samples are obtained by adding tungsten powder to the reference mixture in order to obtain mixtures with low carbon content. This generates a precipitation of Eta-phase in the compounds.

The powder is mixed for 8 hours, dried, and finally compacted with a manual press with a load of 1.8 tons. Successively, a presintering process is carried out; it gives to the pressed powder a consistency similar to the chalk, in order to allow its manipulation and mechanical forming. The process, heating at $900^{\circ} \mathrm{C}$ by an electric oven in a hydrogen atmosphere, determines the liberation of the gas mechanically entrapped in the pressed component, the formation of gas due to internal chemical reactions, and their subsequent evacuation. Preliminary heating at $400-500^{\circ} \mathrm{C}$ allows the removal of a lubricant, used to facilitate uniformity of pressing and reduce friction in the granulometry of the compound. The most common lubricant is paraffin wax. The presintering time depends on the size and shape of the agglomerate. Subsequently, the specimens are placed for $24 \mathrm{~h}$ into the oven, in which the temperature is increased up to $1450^{\circ} \mathrm{C}$, for the sintering process. Table 1 reports the amount of carbon in the WC and the density for each sample, tagged with letters from A (reference sample) to F (sample with poorer carbon content).

Abrasion tests are carried out according to ASTM G9995a standard, by means of a pin-on-disk machine. The specimens, shown in Figure 1, are parallelepipeds with hemispherical ends, $10 \mathrm{~mm}$ in diameter. This shape ensures a continuous contact between the specimens and the abrasive paper, a diamond paper having a grit size of $200 \mu \mathrm{m}$, glued to

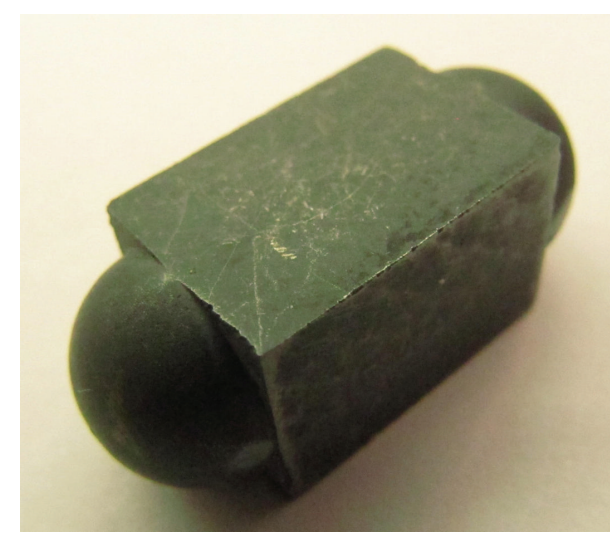

FIGURE 1: Specimen for abrasion tests.

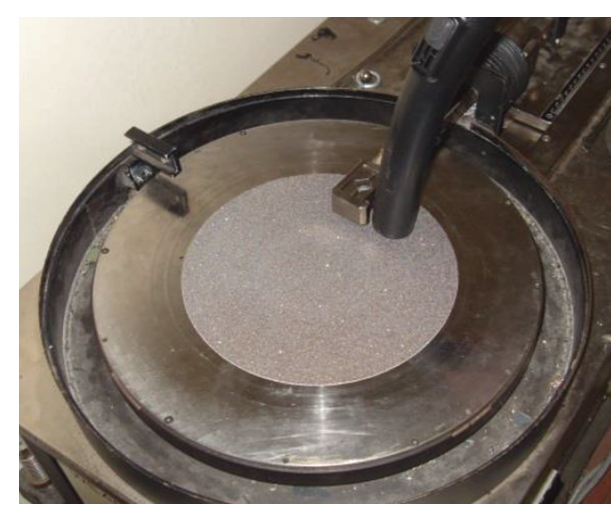

FIgURe 2: Abrasion test.

the disk of the testing machine. Operating conditions of sandblast nozzles in working phase are reproduced; in this regard, a real relative sliding speed $(1 \mathrm{~m} / \mathrm{s})$ and three different loads $(5,10$, and $15 \mathrm{~N})$ are chosen. Different diameters, combined with different rotational speeds (from 100 to $239 \mathrm{rpm}$ ), are chosen in order to describe circular paths with a constant relative sliding speed and on new zones of the abrasive paper for each test. Tests duration of $135 \mathrm{~s}$ guarantees a significant wear track.

Two tests for each load and, then, six tests for each sample are carried out. Both cumulative mass and volume loss at the end of the tests are considered for evaluating the wear. Mass loss is given by the mass difference between the specimen before and after testing, using a precision balance with a sensitivity of $1 \mathrm{mg}$. The volume loss is measured dividing the mass loss by the density, reported in Table 1. Figure 2 shows the execution of an abrasion test, in which it is possible to note an aspirator that cleans the track from the material removed during the test.

In addition to above described tests, hardness and microhardness measures are carried out by means of Rockwell A and micro-Vickers testers, in order to analyze the hardness properties, especially in correspondence with evident singularities, represented by the different shapes and distributions of Eta-phase on the samples. 


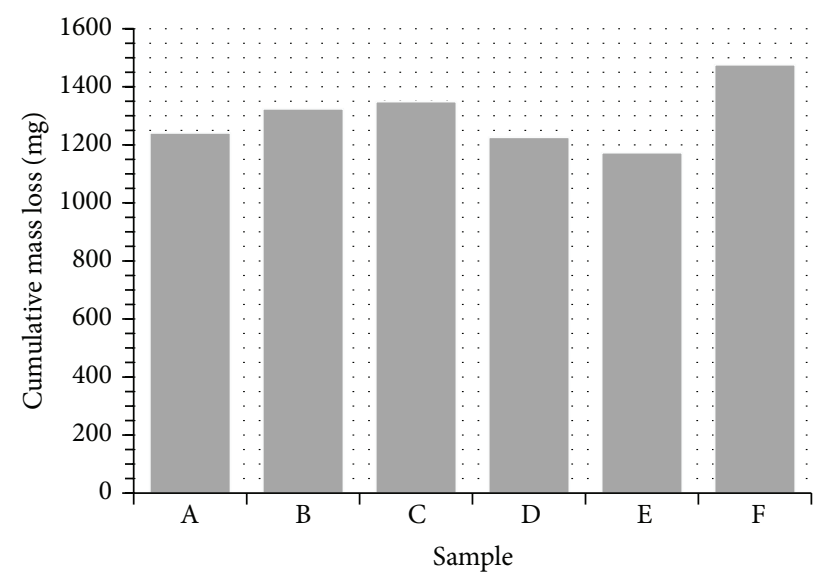

FIGURE 3: Cumulative mass loss.

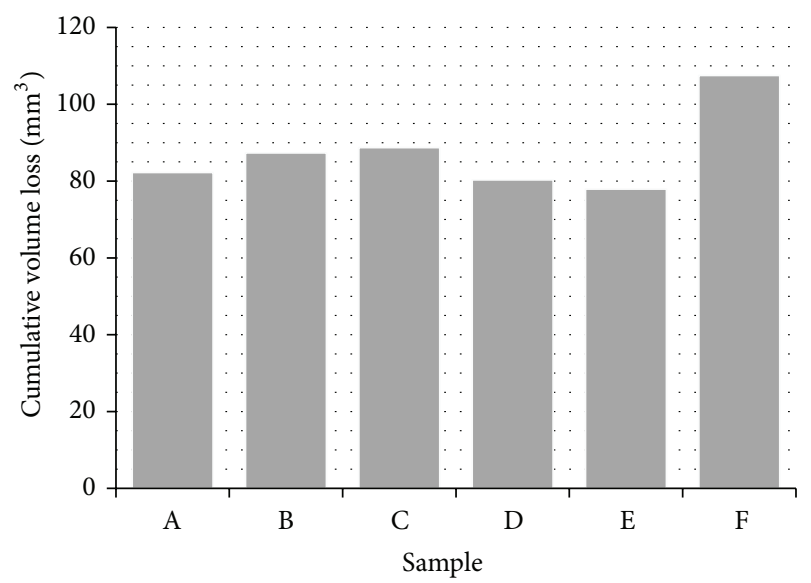

FIgURE 4: Cumulative volume loss.

\section{Results and Discussion}

Figures 3 and 4 show, respectively, the cumulative loss of mass and volume for each sample. Sample E, that is, the mixture with $5.7 \%$ of C, shows the lower loss of mass and volume; this behavior is also verified for each load. Instead, sample F shows the greater mass loss and a still more prominent volume loss, due to a particularly low density. This phenomenon is attributable to the sintering process, which has not reached a complete finalization, and is solvable by increasing the sintering temperature. But, in order to perform analyses compatible with the industrial process of Nashira Hardmetals Company, the sintering temperature is kept equal to $1450^{\circ} \mathrm{C}$ that is one of the stoichiometric mixtures of reference (sample A).

In order to summarize and quantify the differences between the mixtures poor in carbon and the stoichiometric one, their percentage cumulative volume loss variation compared to the reference sample is examined (Figure 5); as a consequence of the above reported observations, sample $\mathrm{F}$ is not considered. As can be seen, the two limit cases are constituted by sample C, with a density of $15.20 \mathrm{~g} / \mathrm{cm}^{3}$ and an amount of carbon equal to $6.00 \%$, that shows the greatest volume loss $(+7.87 \%)$ and sample E, with a density

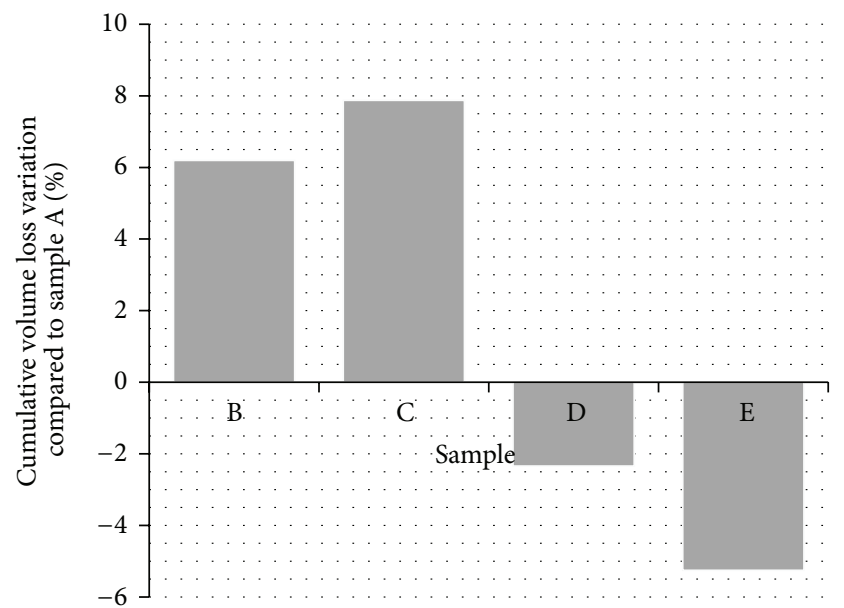

FIgURE 5: Percentage cumulative volume loss variation compared to sample A.

of $15.05 \mathrm{~g} / \mathrm{cm}^{3}$ and an amount of carbon equal to $5.70 \%$, that shows the lowest volume loss $(-5.25 \%)$. However, these results do not allow establishing a direct connection between the carbon content and the wear resistance.

In order to justify the difference in terms of wear, Rockwell A hardness measurements are carried out. In the case of sintered materials, the presence of residual porosity changes the interpretation of the hardness data obtained with the Rockwell traditional tests. The reason is found considering the concept of apparent hardness: with the same microstructure, the hardness is much lower when the porosity is greater, which decreases with the density. Among the various sintered materials, hard metals are a special case: they are characterized by a structure in which there is almost total absence of pores. The tests (five for each sample) are carried out in accordance with ASTM E18-15. The hardness values are in inverse relationship with the depth of the measured impression. The reference mixture has an average hardness of 93.04 HRA, while the nonstoichiometric mixtures have slightly higher values. In fact, the higher value, found for sample B, does not exceed the $0.9 \%$ of sample A hardness. Consequently, these values do not justify the differences in terms of volume loss.

Therefore, micro-Vickers hardness tests are carried out by means of a CV-400 DAT tester on the different areas of the samples, showing or not Eta-phase. In order to identify, in terms of shape and distribution, different species of Etaphase of which to evaluate the microhardness, observations are performed by means of a Zeiss Axioplan 2 upright microscope on metallographic samples, prepared according to ASTM B665-08 (2012) standard; they are etched $5 \mathrm{~s}$ in Murakami's reagent, a solution of 10 g potassium ferricyanide, $10 \mathrm{~g}$ potassium hydroxide, and $100 \mathrm{~mL}$ water.

Figure 6 shows a 1500x magnification of metallographic samples. Only sample A does not show Eta-phase; other ones show Eta-phase in the presumable $\mathrm{M}_{6} \mathrm{C}$ form, since $\mathrm{M}_{12} \mathrm{C}$ is seen only at the substrate/CVD coating interfaces [14].

Sample B shows the formation of Eta-phase in isolated areas and with a star-shaped structure, better observable in 

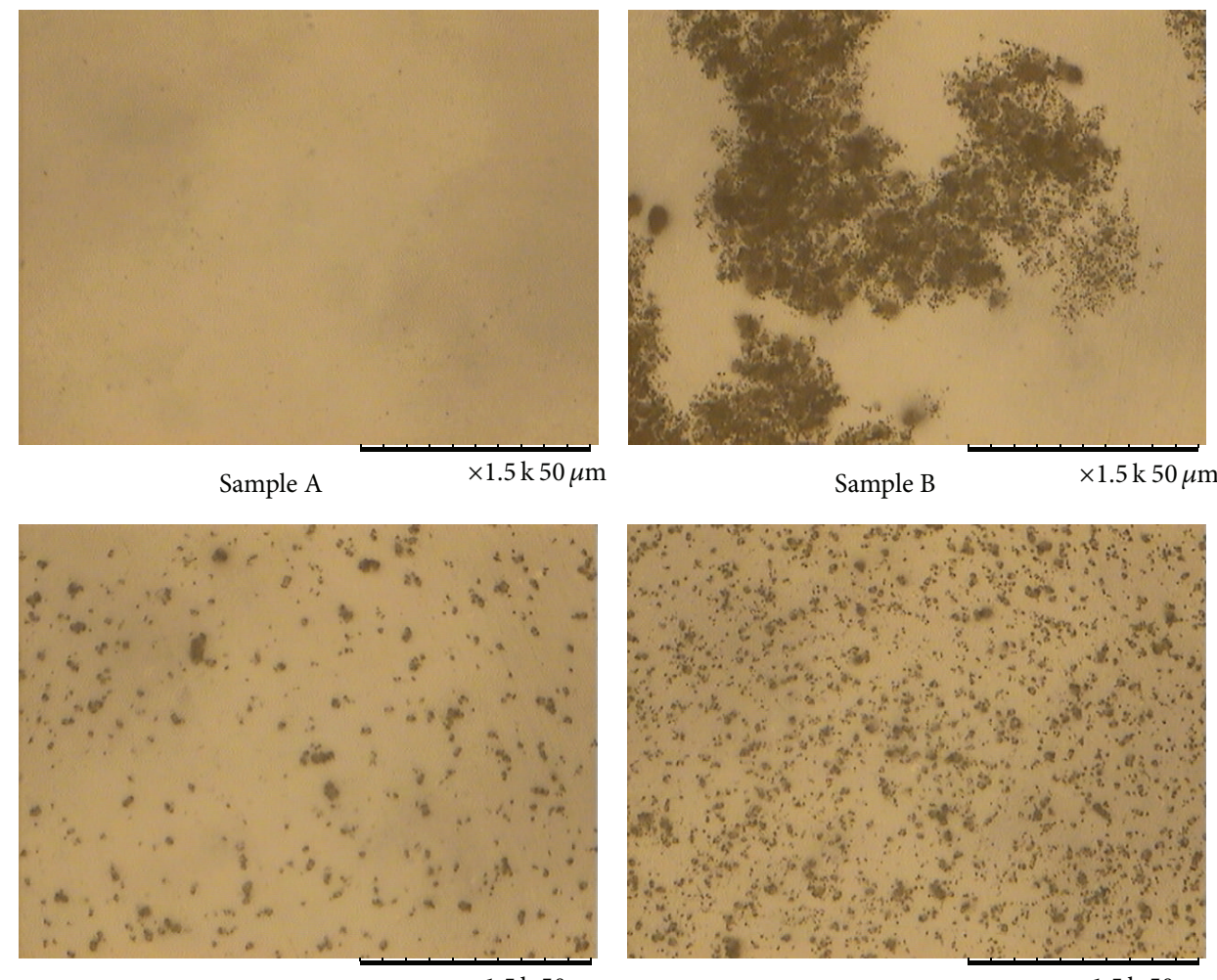

Sample C

$\times 1.5 \mathrm{k} 50 \mu \mathrm{m}$

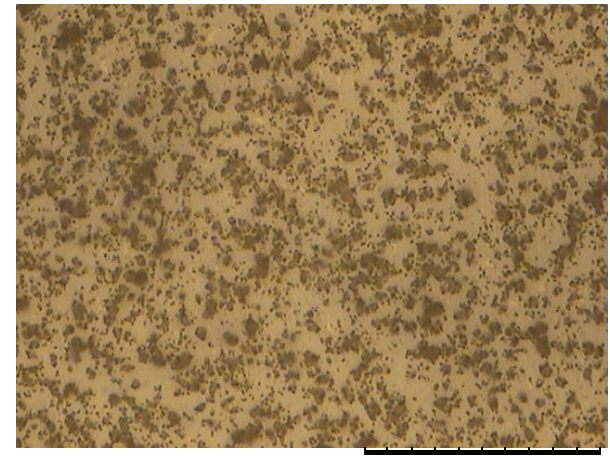

Sample E $\times 1.5 \mathrm{k} 50 \mu \mathrm{m}$

$\times 1.5 \mathrm{k} 50 \mu \mathrm{m}$

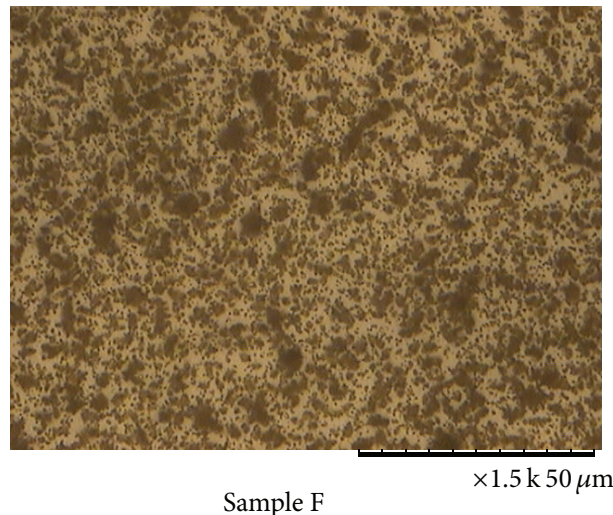

Sample F

FIGURE 6: 1500x magnification of metallographic samples.

a 200x magnification (Figure 7) since it presents great size. This structure is characterized by a low presence on the surface.

Moving away from the stoichiometric value, the formation of a transitional phase characterized by quasi-circular profiles of medium size is observed on sample C; this structure is with a low presence on the surface also.

The Eta-phase formed on the samples from $\mathrm{D}$ to $\mathrm{F}$ shows a quasi-circular shape, both of medium and small size and with a gradually more significant superficial presence.

Micro-Vickers tests are carried out and, concerning the loads used, the requirements according to ASTM C1327-15 standard are considered. The metallographic preparation of the surface is carried out in order to highlight the presence of surface pores and to exclude, as much as possible, their influence, centering with the indenter the area of the surfaces where they apparently do not appear. The microhardness is calculated as the average of five valid measures.

Microhardness measurements are carried out on both samples A and on the different species of Eta-phase of the samples from B to E and, for sample B, also on the free zones of Eta-phase, having larger size than the indenter. The mean values of these measures are reported in Figure 8.

Two important observations are obtained by microhardness measurements, in order to understand the results derived from the abrasion tests. First, regarding sample B, a small carbon deficiency leads to the formation of a very hard Eta-phase type, with a low superficial presence. This phase, immersed in the rest of the material, lowers the macroscopic hardness and this leads to lowering the wear resistance of 


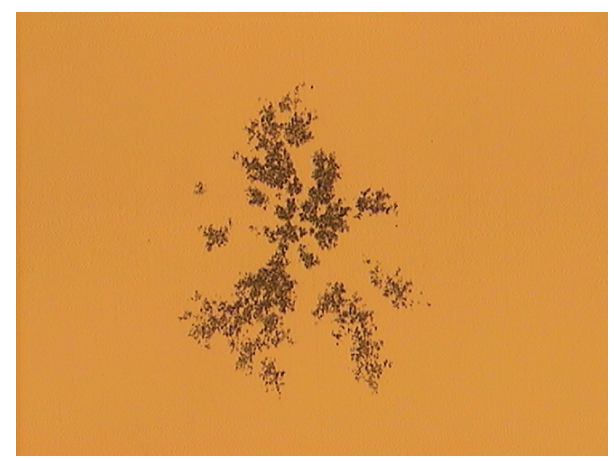

FIgURE 7: 200x magnification of Eta-phase on sample B.

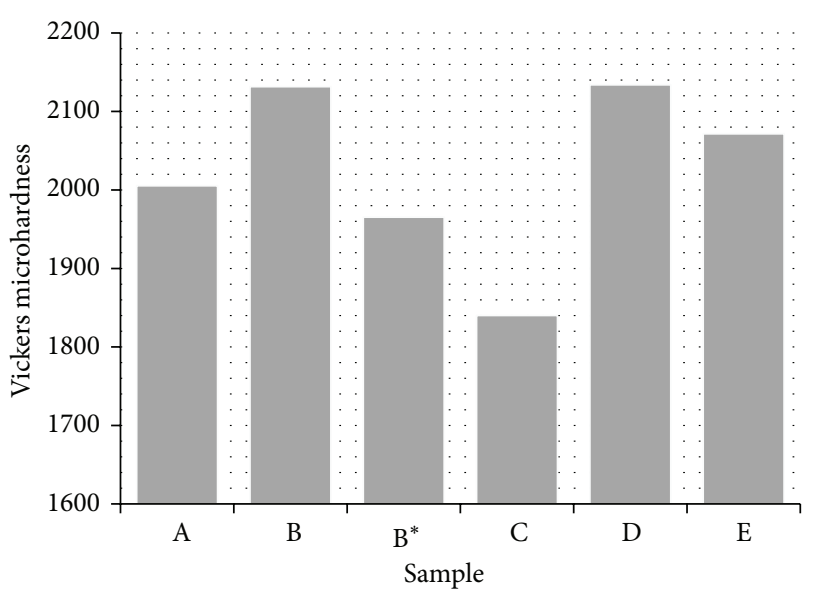

Figure 8: Values of Vickers microhardness $\left(\mathrm{B}^{*}\right.$ is related to sample B free of Eta-phase).

the mixture. Second, from the observation of the measures concerning samples $\mathrm{D}$ and $\mathrm{E}$ it can be seen that the hardness, compared to the one of sample B, decreases, but it is still greater than the reference mixture. The reason is attributable to the presence of a more distributed Eta-phase which leads to a greater wear resistance.

\section{Conclusions}

Eta-phase, always seen as an undesirable presence in the tungsten carbides, may cause, if suitably induced, improvements for the realization of special tools. Sandblast nozzles, not subject to high loads but for which the wear resistance is particularly relevant, are an example. In this regard, the properties of wear resistance of WC-Co carbides in the presence of Eta-phase are analyzed. To understand the effects produced by Eta-phase, six mixtures of carbide with different weight percentages of carbon are prepared and abrasion tests are carried out, using pin-on-disk machine and diamond sandpaper. The tests are carried out by imposing loads and a sliding speed similar to working conditions of sandblast nozzles. The wear is evaluated by the measurement of the mass and volume loss. Moreover, both hardness and microhardness tests and microscopic observations are carried out, in order to interpret the results by abrasion tests.
The tests highlight that wear properties are dependent on both the shape and the distribution of Eta-phase. The samples that show the greatest increase in performance, compared to the stoichiometric one, are those with lower carbon content; these have a gradually smaller Eta-phase size with a larger distribution. On the contrary, worsening of the wear resistance is found for the samples with a carbon percentage slightly lower than the stoichiometric value; these have a distribution of Eta-phase more dense only in certain areas.

Finally, it is useful to observe that, by lowering the percentage of carbon over a certain limit, the correct sintering of the mixture does not allow the standard manufacturing parameters. As future development, higher sintering temperatures may be considered in order to investigate more carbide mixtures.

\section{Conflict of Interests}

The authors declare that there is no conflict of interests regarding the publication of this paper.

\section{Acknowledgments}

This study was carried out with the financial support received from POR Campania 2000/2006, Misura 3.17. The authors thank Dr. Vito Campagnuolo of Nashira Hardmetals Company for substantial collaboration and his suggestions.

\section{References}

[1] S. Norgren, J. Garcia, A. Blomqvist, and L. Yin, "Review. Trends in the P/M hard metal industry," International Journal of Refractory Metals and Hard Materials, vol. 48, pp. 31-45, 2015.

[2] Z. Z. Fang, X. Wang, T. Ryu, K. S. Hwang, and H. Y. Sohn, "Synthesis, sintering, and mechanical properities of nanocrystalline cemented tungsten carbide-a review," International Journal of Refractory Metals and Hard Materials, vol. 27, no. 2, pp. 288-299, 2009.

[3] R. W. Armstrong, "The hardness and strength properties of WC-Co composites," Materials, vol. 4, no. 7, pp. 1287-1308, 2011.

[4] P. J. Blau, "Fifty years of research on the wear of metals," Tribology International, vol. 30, no. 5, pp. 321-331, 1997.

[5] J. Y. Sheikh-Ahmad and J. A. Bailey, "The wear characteristics of some cemented tungsten carbides in machining particleboard," Wear, vol. 225-229, pp. 256-266, 1999.

[6] C. Allen, M. Sheen, J. Williams, and Pugsley V.A, "The wear of ultrafine WC-Co hard metals," Wear, vol. 250-251, no. 1, pp. 604-610, 2001.

[7] G. S. Upadhyaya, "Wear and erosion of cemented carbides," in Cemented Tungsten Carbides: Production, Properties and Testing, pp. 237-248, Noyes Publications, Upper Saddle River, NJ, USA, 1998.

[8] L. Prakash, "Fundamentals and general applications of hardmetals," Comprehensive Hard Materials, vol. 1, pp. 29-90, 2014.

[9] L. Carrino, M. Durante, A. Formisano, A. Langella, F. Capece Minutolo, and A. Caraviello, "Wear behavior of WC-Co carbides with addition of $\mathrm{Cr}_{3} \mathrm{C}_{2}$ and Ni," Key Engineering Materials, vol. 611-612, pp. 444-451, 2014. 
[10] M. Mahmoodan, H. Aliakbarzadeh, and F. Shahri, "Effect of $\mathrm{Cr}_{3} \mathrm{C}_{2}$ and $\mathrm{VC}$ on the mechanical and structural properties of sintered WC-\%10wt Co nano powders," World Journal of Nano Science and Engineering, vol. 3, no. 2, pp. 35-39, 2013.

[11] K. Bonny, P. De Baets, J. Vleugels, S. Huang, O. Van der Biest, and B. Lauwers, "Impact of $\mathrm{Cr}_{3} \mathrm{C}_{2} / \mathrm{VC}$ addition on the dry sliding friction and wear response of WC-Co cemented carbides," Wear, vol. 267, no. 9-10, pp. 1642-1652, 2009.

[12] L. Espinosa, V. Bonache, and M. D. Salvador, "Friction and wear behaviour of WC-Co- $\mathrm{Cr}_{3} \mathrm{C}_{2}-\mathrm{VC}$ cemented carbides obtained from nanocrystalline mixtures," Wear, vol. 272, no. 1, pp. 62-68, 2011.

[13] L. Akesson, "An experimental and thermodynamic study of the Co-W-C system in the temperature range 1470-1700 K," in Science of Hard Materials, R. K. Viswanadham, D. J. Rowcliffe, and J. Gurland, Eds., pp. 71-82, Plenum Press, 1983.

[14] M. N. Haller, "Cemented carbides: metallographic techniques and microstructures," in ASM Handbook Volume 9: Metallography and Microstructures eBook, G. F. Vander Voort, Ed., pp. 513-545, ASM International, Geauga County, Ohio, USA, 2004.

[15] V. K. Sarin, "Morphology of eta phase in cemented WC-Co alloys," Modern Development in Powder Metallurgy, vol. 10, pp. 553-565, 1977.

[16] K. H. Cho, J. W. Lee, and I. S. Chung, "A study on the formation of anomalous large WC grain and the eta phase," Materials Science and Engineering A, vol. 209, no. 1-2, pp. 298-301, 1996.

[17] A. F. Guillermet, "Thermodynamic properties of the Co-W-C system," Metallurgical Transactions A, vol. 20, no. 5, pp. 935956, 1989.

[18] C. B. Pollock and H. H. Stadelmaier, "The eta carbides in the Fe-W-C and Co-W-C systems," Metallurgical Transactions, vol. 1, no. 4, pp. 767-770, 1970.

[19] O. Eso, Z. Z. Fang, and A. Griffo, "Kinetics of cobalt gradient formation during the liquid phase sintering of functionally graded WC-Co," International Journal of Refractory Metals and Hard Materials, vol. 25, no. 4, pp. 286-292, 2007.

[20] G.-H. Lee and S. Kang, "Sintering of nano-sized WC-Co powders produced by a gas reduction-carburation process," Journal of Alloys and Compounds, vol. 419, pp. 281-289, 2006.

[21] V. Bonache, M. D. Salvador, D. Busquets, and E. F. Segovia, "Fabrication of ultrafine and nanocrystalline WC-Co mixtures by planetary milling and subsequent consolidations," Powder Metallurgy, vol. 54, no. 3, pp. 214-221, 2011.

[22] V. Bonache, E. Rayón, M. D. Salvador, and D. Busquets, "Nanoindentation study of WC-12Co hardmetals obtained from nanocrystalline powders: evaluation of hardness and modulus on individual phases," Materials Science and Engineering: A, vol. 527, no. 12, pp. 2935-2941, 2010.

[23] B. Roebuck, A. J. Gant, and M. G. Gee, "Abrasion and toughness property maps for WC/Co hardmetals," Powder Metallurgy, vol. 50, no. 2, pp. 111-114, 2007.

[24] H. So, "Characteristics of wear results tested by pin-on-disc at moderate to high speeds," Tribology International, vol. 29, no. 5, pp. 415-423, 1996. 


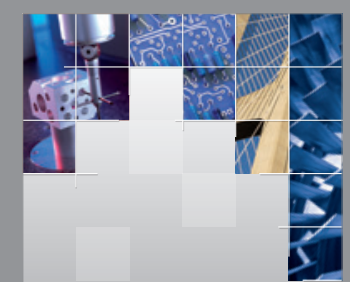

\section{Enfincering}
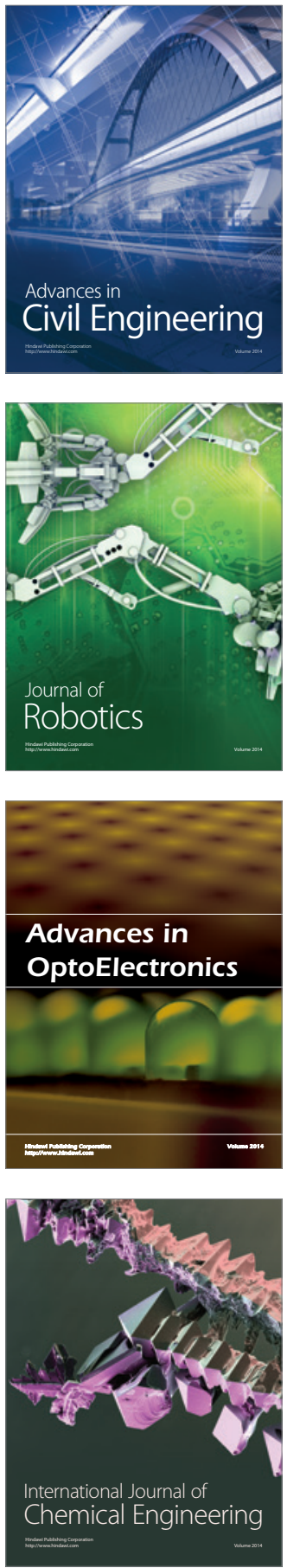

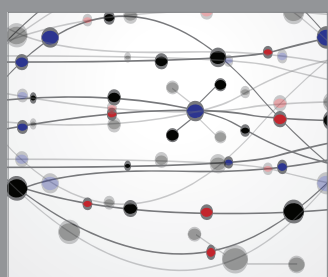

The Scientific World Journal

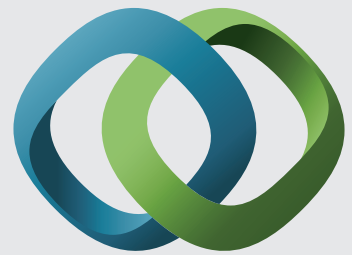

\section{Hindawi}

Submit your manuscripts at

http://www.hindawi.com
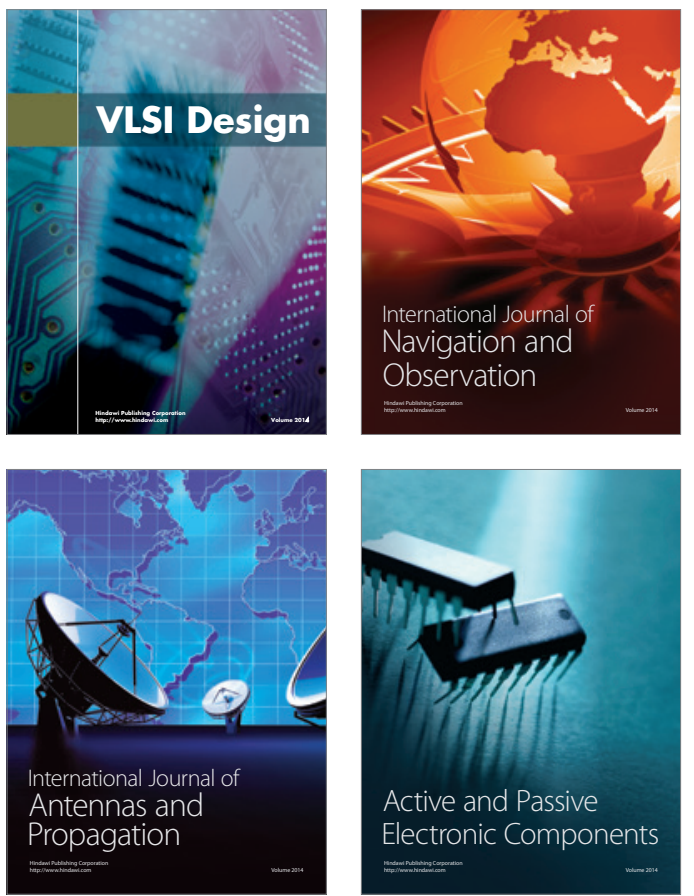
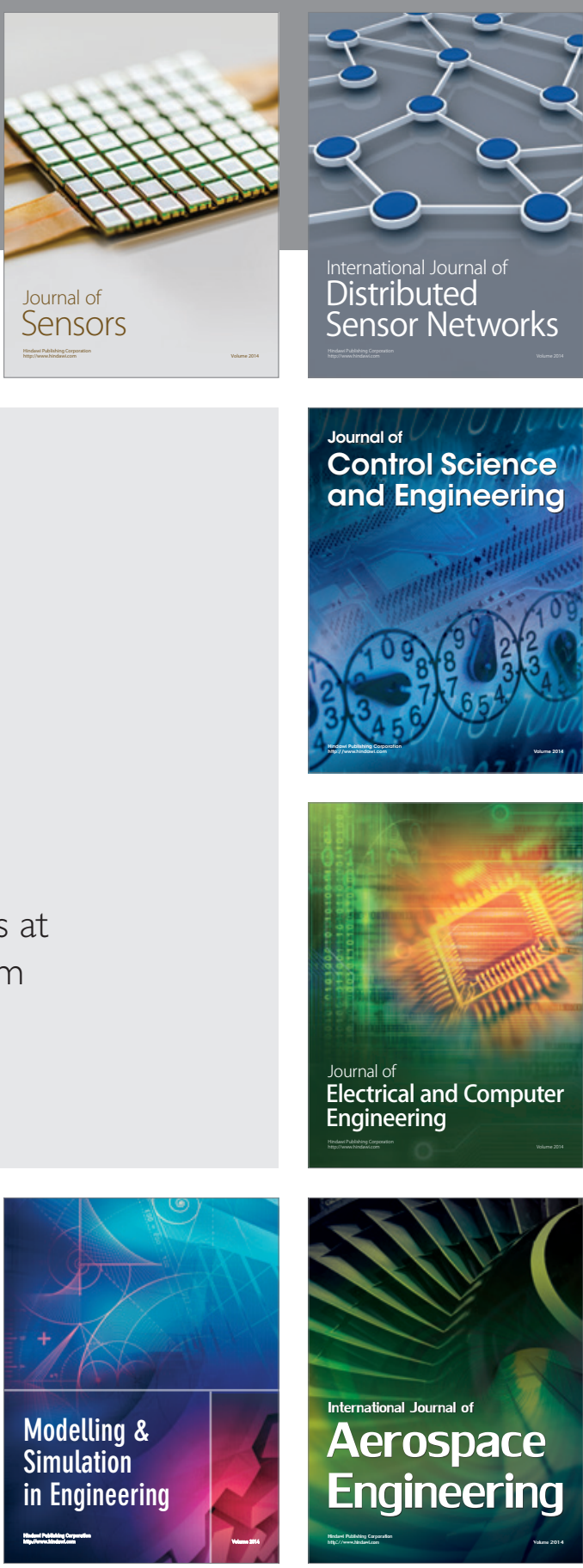

International Journal of

Distributed

Sensor Networks

Journal of

Control Science

and Engineering
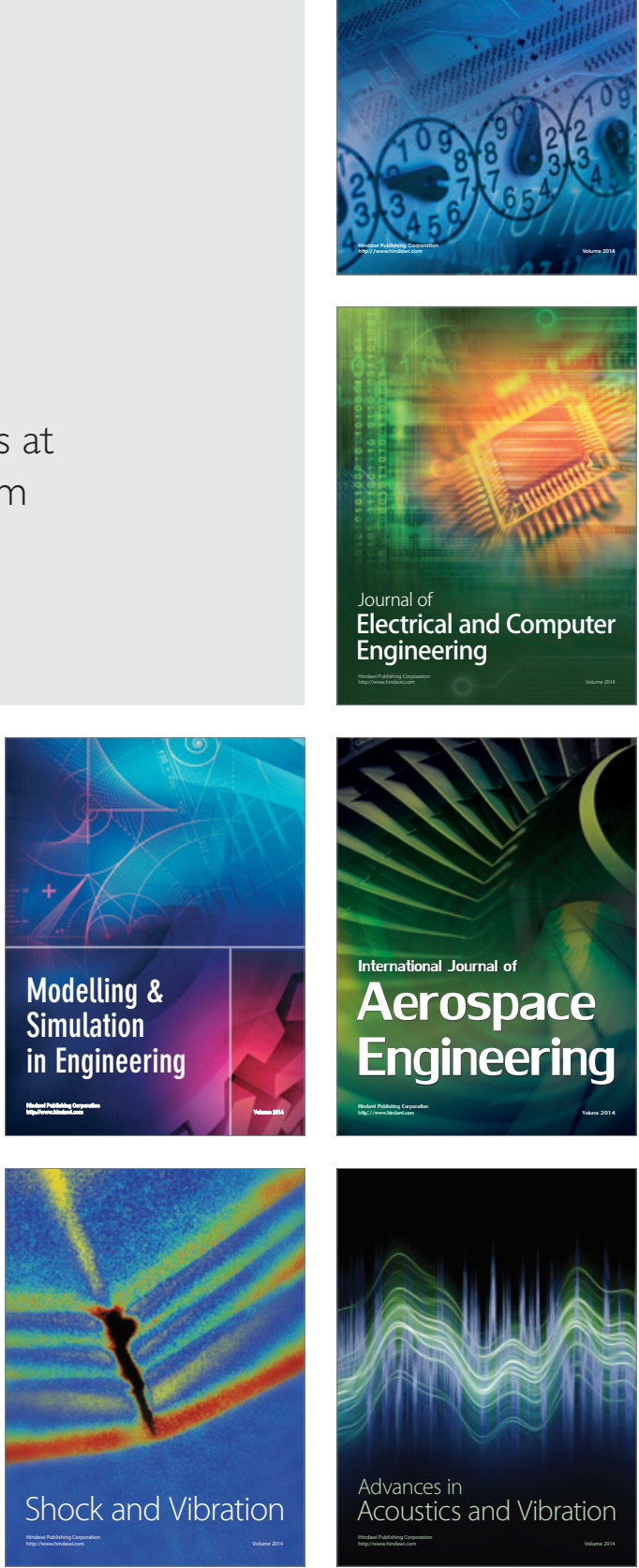